\title{
Riscos e benefícios maternos e neonatais promovidos por partos assistidos na água
}

\author{
Maternal and neonatal risks and benefits promoted by waterbirth
}

Riesgos y beneficios maternos y neonatales promovidos por partos asistidos en el agua

Anny Beatriz Costa Antony de Andrade ${ }^{1 *}$, Andressa Menescal Coelho Azevedo ${ }^{1}$, Katiele de Souza Queiroz ${ }^{1}$, Maria Carolina Fontes Machado ${ }^{1}$, Sabrina Amazonas Farias de Menezes ${ }^{1}$, Bárbara Misslane Cruz Castro?.

\section{RESUMO}

Objetivo: O presente trabalho objetivou analisar os riscos e benefícios, maternos e neonatais, promovidos por partos assistidos na água descritos em produções científicas. Método: Trata-se de uma revisão integrativa, desenvolvida por meio de bases de dados. Foram incluídos 10 artigos de estudos primários publicados no período de 2007 a 2017. Resultados: A diminuição de traumas perineais extensos, redução da dor e diminuição na duração do trabalho de parto e parto foram os principais resultados maternos encontrados. Quanto aos resultados neonatais, estudos comprovam que há melhor adaptação neonatal ao ambiente extrauterino. Considerações finais: A oferta do parto em imersão em água é essencial como alternativa para o alívio da dor e redução do trabalho de parto. É necessária a realização de estudos que avaliem de modo amplo a relação do parto na água por imersão com a integridade perineal e resultados neonatais.

Palavras-chave: Enfermagem Obstétrica, Saúde materna, Trabalho de parto, Parto, Imersão.

\begin{abstract}
Objective: The objective of this study was to analyze maternal and neonatal risks and benefits promoted by assisted births described in scientific productions. Method: This is an integrative review, developed through databases. Ten articles from primary studies published between 2007 and 2017 were included. Results: The reduction of extensive perineal traumas, reduction of pain and decrease in the duration of labor and delivery were the main maternal results. As for neonatal outcomes, studies show that there is better neonatal adaptation to the extrauterine environment. Final considerations: The delivery of water immersion is essential as an alternative for pain relief and reduction of labor. It is necessary to conduct studies that broadly assess the relationship of childbirth in water by immersion with perineal integrity and neonatal outcomes.
\end{abstract}

Keywords: Obstetric Nursing, Maternal Health, Obstetric Labour, Parturition, Waterbirth.

\section{RESUMEN}

Objetivo: El presente trabajo tuvo como objetivo analizar los riesgos y beneficios, maternos y neonatales, promovidos por partos asistidos en el agua descritos en producciones científicas. Método: Se trata de una revisión integrativa, desarrollada a través de bases de datos. Se incluyeron 10 artículos de estudios primarios publicados en el período de 2007 a 2017. Resultados: La disminución de traumas perineales extensos, reducción del dolor y disminución en la duración del trabajo de parto y parto fueron los principales resultados

${ }^{1}$ Universidade Federal do Amazonas, Manaus-AM. * E-mail: antony.beatriz@gmail.com

2 Secretaria de Saúde do Estado do Amazonas, Manaus-AM. 
maternos encontrados. Cuántos a los resultados neonatales, estudios demuestran que hay mejor adaptación neonatal al ambiente extrauterino. Consideraciones finales: La oferta del parto en inmersión en agua es esencial como alternativa para el alivio del dolor y reducción del trabajo de parto. Es necesaria la realización de estudios que evalúen de modo amplio la relación del parto en el agua por inmersión con la integridad perineal y los resultados neonatales.

Palavras-clave: Enfermería Obstétrica, Salud Materna, Trabajo de Parto, Parto, Inmersión.

\section{INTRODUÇÃo}

A utilização da água no trabalho de parto tem sido descrita na literatura desde 1805. Porém, apenas em 1990, o trabalho do pesquisador soviético Igor Tcharkowsky com a imersão de mulheres em banheiras profundas, foi mundialmente reconhecido (ODENT M, 1983; CAVALCANTI AA, 2017).

No Brasil a água tem sido descrita como um método não farmacológico para alívio da dor, ou seja, uma tecnologia leve de cuidado que não demanda o uso de um recurso personalizado, sendo introduzida principalmente para substituir a aplicação de métodos e técnicas invasivas. O uso destes métodos vem sendo amplamente introduzidos nas maternidades do Brasil através das recomendações publicizadas pelos manuais técnicos do Ministério da Saúde e Organização Mundial de Saúde (OMS, 1996; BRASIL, 2017).

O uso da água morna apenas no trabalho de parto está relacionado à promoção do relaxamento muscular e diminuição da sensação dolorosa durante a fase de dilatação e período expulsivo do trabalho de parto (HENRIQUE AJ et al., 2016). Os benefícios do parto na água são descritos na literatura internacional por promover: o aumento da satisfação materna com a experiência do nascimento; maior mobilidade da mulher; diminuição da percepção dolorosa; contrações uterinas mais eficientes; redução do primeiro e do segundo estágio do parto, redução do uso de analgesia, de intervenções no trabalho de parto, da realização de cesariana, de traumas perineais, de episiotomia, de experiências traumáticas de parto, de menor perda de sangue, sem aumento do risco de infecção materna e neonatal e nenhum efeito adverso materno e neonatal observado (MACKEY MM, 2001; ZANETTI-DAELLENBACH RA et al., 2007; CLUETT ER e BURNS E, 2009; MEYER et al., 2010).

Na produção científica brasileira, as publicações sobre parto na água são escassas, com ênfase do tema sobre a frequência de partos assistidos na água em centros de parto normal e em domicílio, sem o registro dos resultados maternos e neonatais (PROGIANTI GM e COSTA RF, 2008; MOUTA RJO e PROGIANTI GM, 2009).

Considerando os benefícios do parto na água e a carência de informações científicas sobre o bem-estar materno e neonatal que encorajem ou desestimulem a sua prática, este estudo teve como objetivo analisar os riscos e benefícios, maternos e neonatais, promovidos por partos assistidos na água descritos em produções científicas.

\section{MÉTODO}

Trata-se de uma revisão integrativa da literatura, método que permite a síntese do conhecimento produzido sobre determinado tema. A finalidade da pesquisa integrativa é de reunir e sintetizar os resultados de pesquisas sobre determinado tema, de forma sistemática e ordenada, permitindo a síntese de estudos publicados e conclusões gerais a respeito de uma área de estudo particular (MENDES KDS et al., 2008).

Para a elaboração do estudo percorreram-se as seguintes etapas, adaptadas: estabelecimento da hipótese, pergunta norteadora: "Quais são as evidências científicas produzidas sobre os benefícios do parto na água, por imersão?"; objetivo da revisão integrativa; estabelecimento de critérios de inclusão e exclusão de artigos; definição das informações a serem extraídas dos artigos selecionados; análise dos resultados; 
discussão e apresentação dos resultados e a última etapa constitui na apresentação da revisão (MENDES KDS et al., 2008).

Os critérios de inclusão dos artigos foram: artigos científicos de estudos primários, publicados no período de 2007 a 2017, nos idiomas português, inglês e espanhol. Foram excluídos artigos por duplicidade.

A coleta de dados foi realizada em outubro de 2017, nas seguintes bases de dados: Literatura LatinoAmericana e do Caribe em Ciências da Saúde (LILACS), Banco de Dados em Enfermagem (BDENF), Medline; PubMed, Web of Science e biblioteca virtual Scientific Eletronic Library Online (SciELO.org). Foram utilizados os seguintes descritores: Trabalho de parto; Parto; Imersão e termos do Medical Subject Headings (MeSH): Labor, obstetric; Parturition; Immersion; Waterbirth.

Para a coleta de dados foram obtidas as seguintes informações: autores, ano de publicação, país de origem do estudo, idioma, local de estudo, população, instrumento de coleta de dados, temperatura da água, resultados maternos e neonatais. O nível de evidência científica foi classificado de acordo com os critérios do Instituto Joanna Briggs (KARINO ME e FELLI VEA, 2012).

Foi confeccionado um fluxograma da pesquisa e seu processo de inclusão dos estudos, baseado no modelo de diagrama PRISMA (MOHER D et al., 2009). Os artigos foram classificados em letras do alfabeto para melhor compreensão dos resultados.

\section{RESULTADOS}

Após o processo de seleção dos estudos encontrados, apenas 11 atenderam aos critérios de inclusão desta pesquisa. O fluxograma desta pesquisa é apresentado na figura 1.

Figura 1. Fluxograma da pesquisa e processo de inclusão dos estudos segundo o PRISMA.

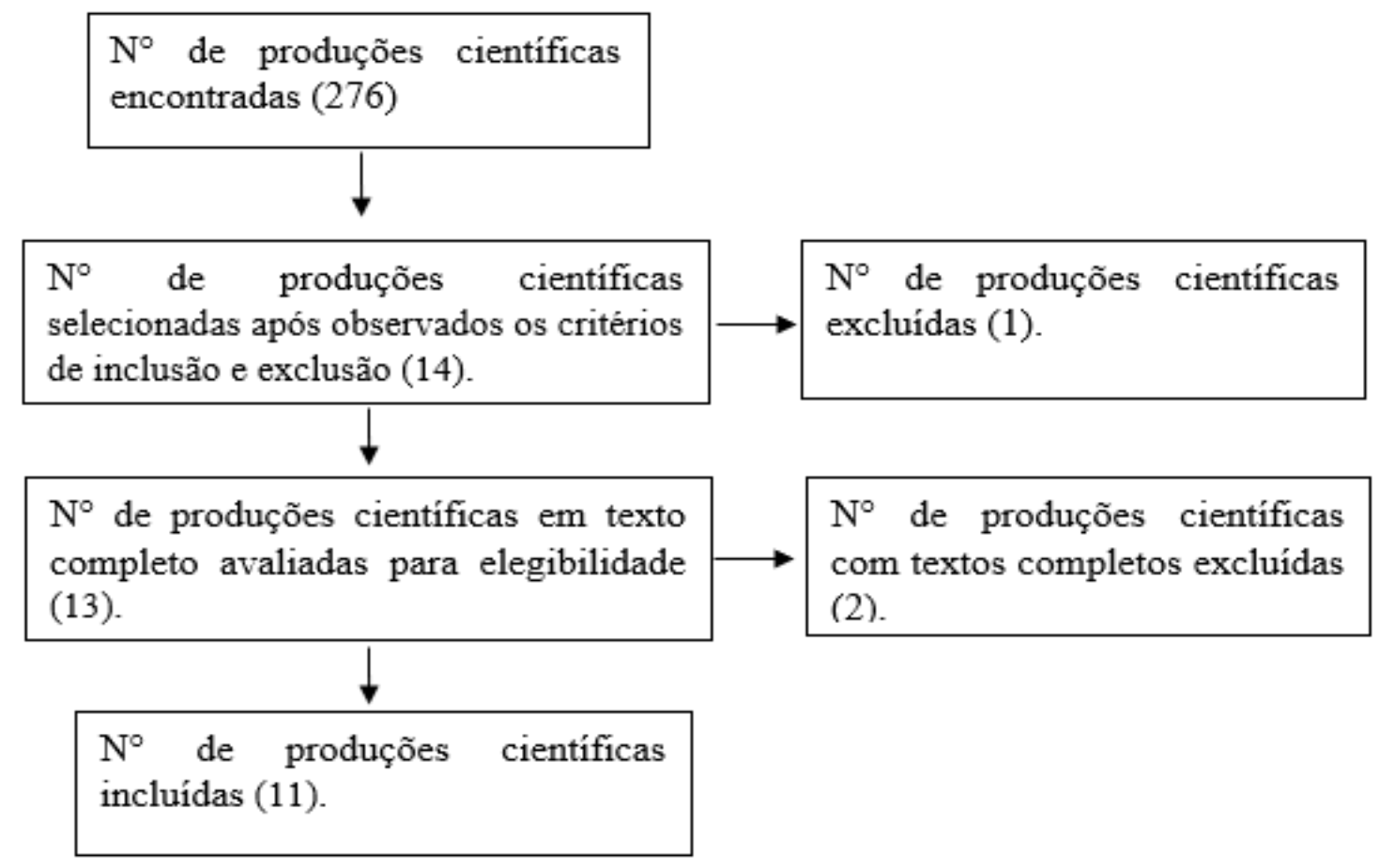

Fonte: Próprio autor, 2018. 
No quadro 1, os estudos foram categorizados segundo os autores, ano de publicação, idioma e país de origem da pesquisa, desenho do estudo, nível de evidência científica, local do estudo, população e instrumento de coleta de dados. Em sua maioria, os estudos realizados foram de coorte, retrospectivo, através da análise de prontuários de parturientes que pariram em imersão e parturientes que pariram de forma convencional, com nível de evidência científica III2.

Quadro 1. Caracterização dos estudos encontrados. Manaus, AM, Brasil, 2018.

\begin{tabular}{|c|c|c|c|c|c|}
\hline $\mathbf{N}$ & Autor (ano) & $\begin{array}{l}\text { País de origem } \\
\text { (idioma) }\end{array}$ & $\begin{array}{l}\text { Desenho do } \\
\text { estudo }\end{array}$ & NE & Local de estudo \\
\hline$A$ & $\begin{array}{l}\text { Zanetti-Daellenbach } \\
\text { RA, et al. (2007) }\end{array}$ & Suíça (inglês) & $\begin{array}{l}\text { Prospectivo/ } \\
\text { Observacional }\end{array}$ & III3 & $\begin{array}{l}\text { Hospital Universitário } \\
\text { Feminino }\end{array}$ \\
\hline B & $\begin{array}{l}\text { Meyer SL, et al. } \\
(2010)\end{array}$ & $\begin{array}{l}\text { Estados Unidos } \\
\text { (inglês) }\end{array}$ & Quanti-qualitativo & IV & $\begin{array}{l}\text { Pesquisa realizada através } \\
\text { de e-mail }\end{array}$ \\
\hline C & $\begin{array}{l}\text { Cortes E, et al. } \\
(2011)\end{array}$ & Reino Unido (inglês) & $\begin{array}{l}\text { Coorte/ } \\
\text { Retrospectivo }\end{array}$ & III2 & $\begin{array}{l}\text { Hospital com centro de } \\
\text { parto }\end{array}$ \\
\hline D & $\begin{array}{l}\text { Mollamahmutoğlu L, } \\
\text { et al (2011) }\end{array}$ & Turquia (inglês) & $\begin{array}{l}\text { Ensaio clínico } \\
\text { prospectivo }\end{array}$ & II & $\begin{array}{l}\text { Hospital de Educação e } \\
\text { Pesquisa em Saúde da } \\
\text { Mulher }\end{array}$ \\
\hline$E$ & $\begin{array}{l}\text { Menakaya U, et al. } \\
(2013)\end{array}$ & Austrália (inglês) & $\begin{array}{l}\text { Coorte/ } \\
\text { Retrospectivo }\end{array}$ & III2 & Hospital de Bankstown \\
\hline $\mathrm{F}$ & $\begin{array}{l}\text { Dahlen HG, et al. } \\
(2013)\end{array}$ & Austrália (inglês) & $\begin{array}{l}\text { Descritivo/ } \\
\text { Transversal }\end{array}$ & IV & $\begin{array}{l}\text { Centro de parto em } \\
\text { Sydney }\end{array}$ \\
\hline G & Liu Y, et al. (2014) & China (inglês) & Comparativo & III3 & $\begin{array}{l}\text { Obstetrical Department of } \\
\text { Sun Yat-sen Memorial } \\
\text { Hospital }\end{array}$ \\
\hline $\mathrm{H}$ & $\begin{array}{l}\text { Mckenna JA, et al. } \\
(2014)\end{array}$ & Reino Unido (inglês) & $\begin{array}{l}\text { Qualitativo/ } \\
\text { Fenomenológico }\end{array}$ & IV & $\begin{array}{l}\text { Unidade escocesa liderada } \\
\text { por parteira }\end{array}$ \\
\hline I & $\begin{array}{l}\text { Henderson J, et al. } \\
\text { (2014) }\end{array}$ & Itália (inglês) & $\begin{array}{l}\text { Prospectivo/ } \\
\text { Observacional }\end{array}$ & III3 & $\begin{array}{l}19 \text { unidades obstétricas } \\
\text { italianas }\end{array}$ \\
\hline$J$ & $\begin{array}{l}\text { Lim KMX, et al. } \\
(2016)\end{array}$ & Singapore (inglês) & $\begin{array}{l}\text { Coorte/ } \\
\text { Retrospectivo }\end{array}$ & III2 & $\begin{array}{l}\text { Hospital Universitário } \\
\text { Nacional }\end{array}$ \\
\hline K & $\begin{array}{l}\text { Bovbjerg ML, et al. } \\
(2016)\end{array}$ & $\begin{array}{l}\text { Estados } \\
\text { (inglês) }\end{array}$ & $\begin{array}{l}\text { Coorte/ } \\
\text { Retrospectivo }\end{array}$ & III2 & $\begin{array}{l}\text { Banco de dados Midwives } \\
\text { Alliance of North America } \\
\text { Statistics Project }\end{array}$ \\
\hline
\end{tabular}

Fonte: Próprio autor, 2018.

No quadro 2, foram dispostos os principais resultados maternos e neonatais encontrados nos estudos, bem como a temperatura da água. Dentre os principais resultados maternos identificou-se a redução da taxa de episiotomias e traumas perineais extensos $(D, E, H, I, J)$, maior relaxamento durante o trabalho de parto e redução no uso de analgésicos $(A, B, D, G, H)$, redução na duração do trabalho de parto $(C, D, J)$. 
Quadro 2. Principais resultados maternos e neonatais. Manaus, AM, Brasil, 2018.

\begin{tabular}{|c|c|c|c|}
\hline \multirow[t]{2}{*}{$\mathbf{N}$} & \multirow{2}{*}{$\begin{array}{l}\mathrm{T}^{\star} \quad \mathrm{da} \\
\text { água }\left({ }^{\circ} \mathrm{C}\right)\end{array}$} & \multicolumn{2}{|c|}{ Desfechos } \\
\hline & & Maternos & Neonatais \\
\hline A & $\begin{array}{l}\text { Não } \\
\text { especifica } \\
\text { do }\end{array}$ & $\begin{array}{l}\text { Menor uso de analgésicos, menor duração do } \\
\text { trabalho de parto e episiotomias. }\end{array}$ & $\begin{array}{l}\text { Sem diferenças entre escores de Apgar, } \\
\text { sem admissão do recém-nato em } \\
\text { unidades de terapia intensiva. }\end{array}$ \\
\hline $\mathrm{B}$ & $\begin{array}{l}\text { Não } \\
\text { especifica } \\
\text { do }\end{array}$ & $\begin{array}{l}\text { Menor uso de analgésicos, maior relaxamento } \\
\text { da mulher, pouca redução no volume de } \\
\text { perda sanguínea e nos traumas perineais. }\end{array}$ & $\begin{array}{l}\text { Melhor adaptação neonatal após o } \\
\text { nascimento. }\end{array}$ \\
\hline C & $\begin{array}{l}\text { Não } \\
\text { especifica } \\
\text { do }\end{array}$ & $\begin{array}{l}\text { Redução do } 2^{\circ} \text { estágio do trabalho de parto e } \\
\text { aumento de lacerações de } 3^{\circ} \text { grau. Maior } \\
\text { satisfação com o parto. }\end{array}$ & Não especificado \\
\hline $\mathrm{D}$ & $\begin{array}{l}37^{\circ} \mathrm{C} \\
37,5^{\circ} \mathrm{C}\end{array}$ & $\begin{array}{l}\text { Redução da dor, do } 2^{\circ} \text { e } 3^{\circ} \text { estágios do } \\
\text { trabalho de parto e de intervenções. Maior } \\
\text { índice de lacerações. }\end{array}$ & $\begin{array}{l}\text { Sem diferenças entre escores de Apgar, } \\
\text { sem admissão do recém-nato em } \\
\text { unidades de terapia intensiva. }\end{array}$ \\
\hline$E$ & 36 e $37^{\circ} \mathrm{C}$ & $\begin{array}{l}\text { Sem diferença significativa no } 1^{\circ} \text { e } 2^{\circ} \text { estágios } \\
\text { do trabalho de parto e hemorragia puerperal, } \\
\text { entre os grupos. Sem registros de traumas } \\
\text { perineais extensos e episiotomias. }\end{array}$ & $\begin{array}{l}\text { Maior incidência para escore de Apgar } \\
<7 \quad\left(1^{\circ} \text { minuto }\right) \text {, houve significativa } \\
\text { admissão em unidades de cuidados } \\
\text { especiais e menor taxa de permanência } \\
\text { hospitalar. }\end{array}$ \\
\hline $\mathrm{F}$ & $\begin{array}{l}\text { Não } \\
\text { especifica } \\
\text { do }\end{array}$ & $\begin{array}{l}\text { Menor incidência de trauma perineal e de } \\
\text { hemorragia puerperal. }\end{array}$ & $\begin{array}{l}\text { Maior incidência de escore de Apgar }<7 \\
\left(1^{\circ} \text { e } 5^{\circ} \text { minuto }\right) \text {. }\end{array}$ \\
\hline G & 35 e $38^{\circ} \mathrm{C}$ & $\begin{array}{l}\text { Redução da dor, menor taxa de cesáreas e } \\
\text { infecção urinária no puerpério. A duração do } \\
\text { trabalho de parto e sangramento foi } \\
\text { semelhante em ambos os grupos. }\end{array}$ & $\begin{array}{l}\text { Sem diferenças entre escores de Apgar. } \\
\text { Transferência de neonatos de parto na } \\
\text { água para unidades pediátricas }(n=4) \text {. }\end{array}$ \\
\hline $\mathrm{H}$ & $\begin{array}{l}\text { Não } \\
\text { especifica } \\
\text { do }\end{array}$ & $\begin{array}{l}\text { Relaxamento durante o trabalho de parto, } \\
\text { melhor recuperação pós-parto, menos } \\
\text { desordens psicológicas, maior atenção e } \\
\text { cuidado com os bebês. }\end{array}$ & Não especificado. \\
\hline I & $\begin{array}{l}\text { Não } \\
\text { especifica } \\
\text { do }\end{array}$ & $\begin{array}{l}\text { Nulíparas de parto na água apresentaram } \\
\text { menos episiotomias. Casos raros de trauma } \\
\text { perineal extenso e hemorragia pós-parto. }\end{array}$ & Não houve resultados adversos graves. \\
\hline$J$ & 35 a $37^{\circ} \mathrm{C}$ & $\begin{array}{l}\text { Menor tempo de trabalho de parto, de } \\
\text { hemorragias e episiotomias. Maior } \\
\text { quantidade de períneos íntegros. Casos } \\
\text { isolados de retenção placentária. }\end{array}$ & $\begin{array}{l}\text { Sem diferenças entre escores de Apgar. } \\
\text { A taquipneia transitória do recém- } \\
\text { nascido apresentou maior incidência em } \\
\text { partos convencionais. }\end{array}$ \\
\hline $\mathrm{K}$ & $\begin{array}{l}\text { Não } \\
\text { especifica } \\
\text { do }\end{array}$ & $\begin{array}{l}\text { Diminuição da hospitalização após o parto, } \\
\text { aumento de chances de trauma perineal. Sem } \\
\text { associações do parto na água com aumento } \\
\text { de risco de infecção materna. }\end{array}$ & $\begin{array}{l}\text { Menor incidência de transferência } \\
\text { neonatal para hospital e admissão em } \\
\text { unidade de terapia intensiva. Melhor } \\
\text { adaptação ao meio extrauterino. }\end{array}$ \\
\hline
\end{tabular}

Fonte: Próprio autor, 2018. *Temperatura 
Não houve diferença significativa para a redução da hemorragia puerperal $(B, E, F, I, J)$. Houve casos isolados de retenção placentária. Houve a melhora da disposição para o exercício da maternidade, entre as mulheres que optaram pelo parto na água, com a redução de quadros de distúrbios psicológicos $(H)$.

Quanto aos resultados neonatais, não houve diferenças significativas entre o escore de Apgar de recémnatos de parto na água de parto convencional (D, E, F, G, I, J). Observou-se aumento nas taxas de contato pele a pele e vínculo do binômio mãe-bebê, com melhor evolução da adaptação da criança ao ambiente extrauterino $(B, K)$. Houve casos de transferência neonatal para unidades de cuidados pediátricos, porém com menor incidência que os recém-nascidos de partos convencionais $(E, G, K)$. Os recém-nascidos de parto na água apresentaram menor taxa de permanência hospitalar, bem como o menor desenvolvimento de condições como a taquipneia transitória do recém-nascido $(\mathrm{J}, \mathrm{K})$.

\section{DISCUSSÃO}

Os estudos sugerem que quando o parto ocorre na água a duração dos estágios do trabalho de parto pode ser reduzida de forma significativa. Pesquisadores identificaram diferenças principalmente no período expulsivo dos trabalhos de parto acompanhados, com redução significativa desse período no grupo de mulheres que pariram dentro da água (CORTES E et al., 2011; MOLLAMAHMUTOĞLU L et al., 2012). O período de dequitação foi mais curto no parto na água, trazendo benefícios como a redução da perda sanguínea neste período (MOLLAMAHMUTOǦLU L et al., 2012).

O uso da imersão em água no trabalho de parto, mostrou-se importante para a redução significativa do período de dilatação e expulsivo do trabalho de parto (ZANETTI-DAELLENBACH RA et al., 2007). Esta redução foi encontrada em outros estudos, com até 32 minutos de diferença, quando comparadas às mulheres de parto convencional com as mulheres de parto na água (CLUETT ER e BURNS E, 2009). Além da influência da hidroterapia na duração do trabalho de parto, a mesma também pode ser utilizada como recurso para 0 alívio da dor (CORTES E et al., 2011).

Os traumas perineais estão relacionados à episiotomia e lacerações que ocorrem durante o trabalho de parto. Um dos estudos analisados afirma que a ocorrência de lacerações perineais no parto na água foi mais incidente, porém de grau menos severo se comparado aos grupos com analgesia peridural e de parto convencional, além do parto na água proporcionar às parturientes menos intervenções obstétricas como indução e episiotomia (MOLLAMAHMUTOǦLU L et al., 2012). Outros estudos também trazem em seus resultados a pouca redução de trauma perineal, bem como o aumento da incidência de lacerações de $3^{\circ}$ grau relacionado à redução do $2^{\circ}$ estágio do trabalho de parto, cujo encurtamento do tempo afeta diretamente na adaptação da musculatura perineal para as forças exercidas na descida do concepto (MEYER SL et al., 2010; CORTES E et al., 2011; BOVBJERG ML et al., 2016).

Por outro lado, dois estudos afirmam que, em comparação aos grupos intervenção e controle, houve menor incidência de episiotomia e trauma perineal, permanecendo a maioria das mulheres com o períneo íntegro (DAHLEN HG et al., 2013; LIM KMX et al., 2016). Em se tratando de nulíparas, as intervenções obstétricas tendem a se mostrar mais presentes no avançar do trabalho de parto. Entretanto, o grupo de nulíparas apresentou lacerações perineais espontâneas de segundo grau, sem ocorrência de episiotomia (CORTES E et al., 2011; HENDERSON J et al., 2014). Quando comparados os grupos de nascimento na água e parto convencional, os achados foram significantes, não havendo registros de traumas perineais $\mathrm{e}$ episiotomia no grupo do parto na água (DAHLEN HG et al., 2013).

A ocorrência de infecção e hemorragia puerperal relacionadas ao parto na água foram evidenciadas em alguns estudos, embora descritas como condições raras, observadas em casos isolados (HENDERSON $\mathrm{J}$ et al., 2014; BOVBJERG ML et al., 2016). O sangramento eliminado no período pós-parto foi semelhante ao de partos convencionais (MENAKAYA U et al., 2013; LIU Y et al., 2014). Portanto, de forma a evitar o agravo à saúde materna, recomenda-se que o parto na água seja oferecido às mulheres que apresentem chances mínimas ao desenvolvimento de infecções puerperais e hemorragias pós-parto. 
Mulheres incentivadas a adotar posições não supinas, estimuladas a exercerem autonomia e harmonia no trabalho de parto, demonstraram interesse pelo meio aquático durante o processo de parturição. Foi observado o relaxamento da mulher enquanto permaneceu em contato com a água morna, reduzindo a necessidade de prescrição de fármacos analgésicos (ZANETTI-DAELLENBACH RA et al., 2007; MOLLAMAHMUTOǦLU L et al., 2012; McKENNA JÁ e SYMON AG, 2014). A associação da imersão em água morna com a penumbra reduz significantemente a quantidade de trabalhos de partos laboriosos (ODENT M, 1983). Dessa forma, reitera-se a importância da oferta do banho de imersão como prática não farmacológica para alívio da dor durante o primeiro estágio do trabalho de parto, mesmo que a mulher opte pelo parto de forma convencional.

É necessário esclarecer que o ambiente aquático, para a parturição, deve ser oferecido preferencialmente a mulheres que possuem conhecimento prévio sobre o trabalho de parto e parto na água, riscos e benefícios do mesmo (HENDERSON $J$ et al., 2014). Recomenda-se que os partos na água sejam indicados apenas a mulheres com gestações classificadas como baixo risco (HENDERSON J et al., 2014; LIM KMX et al., 2016). Portanto, torna-se necessária a associação da prática com protocolos de classificação de risco em obstetrícia, de forma a minimizar os danos à saúde materna e neonatal.

Embora a produção científica sobre o parto na água desmistifique resultados negativos e recomende a sua prática, a aplicabilidade do mesmo no cenário das maternidades brasileiras ainda não é uma realidade existente, uma vez que não há banheira instalada na maioria dos centros de parto normal. Considerando os resultados positivos sobre o bem-estar materno, é importante que haja o encorajamento de reestruturação física das instituições hospitalares (GAYESKI ME e BRUGGEMANN OM, 2010).

Os desfechos de neonatos em partos na água são amplamente discutidos no meio científico. Na maioria das produções científicas identificadas, concluiu-se que não há influência do parto na água no escore de Apgar. Recém-nascidos de uma análise de 6.534 prontuários de partos assistidos na água, não apresentaram maiores riscos para o desenvolvimento de resultados adversos, apresentando melhor evolução que neonatos de partos assistidos fora da água (BOVBJERG ML et al., 2016). A ocorrência do parto na água é contraindicada quando existe o risco de sofrimento fetal, principalmente na presença de padrões anormais de frequência cardíaca fetal (ZANETTI-DAELLENBACH RA et al., 2007).

Salienta-se que a monitorização da frequência cardíaca fetal é importante tanto para partos na água como fora da água, e seu rigor durante o trabalho de parto deve ser observado, de forma a promover a saúde neonatal.

Reitera-se que o parto na água, deve conduzido por equipe multiprofissional capacitada, onde o ambiente ofereça o suporte assistencial e de reanimação adequados, e esteja localizado próximo à um ambiente hospitalar, de forma a oferecer o suporte materno-neonatal adequados, evitando intercorrências e promovendo experiências exitosas para as mulheres, seus familiares e equipe de saúde.

\section{CONSIDERAÇÕES FINAIS}

Com base nos achados desta pesquisa, pode-se concluir que o parto na água representa uma opção segura para a mulher que o deseje, devendo ser respeitada a autonomia e a escolha feminina sobre o local e ambiente de parto. Em se tratando dos resultados maternos, identificou-se nos estudos as evidências de que a imersão é capaz de reduzir a dor, encurtar a duração do trabalho de parto, reduzir os traumas perineais, a ocorrência de cesáreas, a prática da episiotomia e o índice de infecções. Embora poucos estudos tenham avaliado os resultados neonatais em partos na água, não existe evidência científica clara que o contraindique, com a exceção nas situações em que há padrões de frequência cardíaca fetal anormais. Portanto há necessidade do desenvolvimento de estudos, como ensaios clínicos randomizados, para avaliar de modo mais eficaz os resultados maternos e neonatais. 


\section{REFERÊNCIAS}

1. BOVBJERG ML, CHEYNEY M, EVERSON C. Maternal and Newborn Outcomes Following Waterbirth: The Midwives Alliance of North America Statistics Project, 2004 to 2009 Cohort. J Midwifery Women's Heal, jan 2016; 61(1): 11-20.

2. BRASIL. Ministério da Saúde. Diretrizes Nacionais de assistência ao parto normal: versão resumida [recurso eletrônico]. Brasília, 2017.

3. CAVALCANTI AA. O cinema documentário como tática: uma revisão da produção filmica pela humanização da assistência ao parto (1940-2017). Quaderns-e de l'Institut Català d'Antropologia, 2017; 22(1): 105-121.

4. CLUETT ER, BURNS E. Immersion in water in labour and birth. Cochrane Database of Systematic Reviews, apr 2009; 2.

5. CORTES E, BASRA R, KELLEHER CJ. Waterbirth and pelvic floor injury: A retrospective study and postal survey using ICIQ modular long form questionnaires. Eur J Obstet Gynecol Reprod Biol, mar 2011; 155(1): 27-30.

6. DAHLEN HG, DOWLING H, TRACY $M$ et al. Maternal and perinatal outcomes amongst low risk women giving birth in water compared to six birth positions on land. A descriptive cross sectional study in a birth centre over 12 years. Midwifery, jul 2013; 29(7): 759-64.

7. GAYESKI ME, BRÜGGEMANN OM. Métodos não farmacológicos para alívio da dor no trabalho de parto: uma revisão sistemática. Texto contexto - enferm, oct/dec 2010; 4:774-82.

8. HENDERSON J, BURNS EE, REGALIA AL, et al. Labouring women who used a birthing pool in obsteric units in Italy: prospective observational study. BMC Pregnancy Childbirth, jan 2014; 14:17.

9. HENRIQUE AJ, GABRIELLONI MC, CAVALCANTI ACV et al. Hidroterapia e bola suiça no trabalho de parto: ensaio clínico randomizado. Acta Paul Enferm, 2016; 29(6): 686-92.

10. KARINO ME, FELLI VEA. Enfermagem Baseada em Evidências: Avanços e inovações em revisões sistemáticas. Cienc Cuid Saude, 2012; 11(suplem.): 11-5.

11. LIM KMX, TONG PSY, CHONG YS. A comparative study between the pioneer cohort of waterbirths and conventional vaginal deliveries in an obstetrician-led unit in Singapore. Taiwan J Obstet Gynecol, jun 2016; 55(3): 363-7.

12. LIU Y, LIU Y, HUANG $X$ et al. A comparison of maternal and neonatal outcomes between water immersion during labor and conventional labor and delivery. BMC Pregnancy Childbirth, may 2014; $1-7$.

13. MACKEY MM. Use of Water in Labor and Birth. Clin Obstet Gynecol, dec $2001 ; 44(4)$.

14. MCKENNA JA, SYMON AG. Water VBAC: Exploring a new frontier for women's autonomy. Midwifery, jan 2014; 30(1): e20-5.

15. MENAKAYA U, ALBAYATI S, VELLA E et al. A retrospective comparison of water birth and conventional vaginal birth among women deemed to be low risk in a secondary level hospital in Australia. Women and Birth, jun 2013; 26(2): 114-8.

16. MENDES KDS, SILVEIRA RC DE CP, GALVÃO CM. Revisão integrativa: método de pesquisa para a incorporação de evidências na saúde e na enfermagem. Texto contexto - enferm, oct/dec 2008; 17(4): 758-64

17. MEYER SL, WEIBLE CM, WOEBER K. Perceptions and Practice of Waterbirth: A Survey of Georgia Midwives. J Midwifery Women's Heal, jan/feb 2010; 55(1): 55-9.

18. MOHER D, LIBERATI A, TETZLAFF J et al. Preferred reporting items for systematic reviews and meta-analyses: the PRISMA statement. PLoS Med, 2009 Jul; 6(7): e1000097.

19. MOLLAMAHMUTOǦLU L, MORALOĞLU Ö, ÖZYER Ş et al. Suda doğumun, travay, doğum ve yenidoğan üzerine etkileri ve epidural analjezi ile normal doğum ve konvansiyonel vajinal doğum ile karşi\{dotless\}laşti\{dotless\}ri\{dotless\}lmasi\{dotless\}. J Turkish Ger Gynecol Assoc, mar 2012; 13(1): 45-9.

20. MOUTA RJO, PROJIANTI JM. Estratégias de luta das enfermeiras da maternidade Leila Diniz para implantação de um modelo humanizado de assistência ao parto. Texto contexto - enferm, oct-dec 2009; 18(4): 731-40.

21. ODENT M. Birth Under Water. The Lancet, dec 1983; 322(8365-8366): 1476-7.

22. ORGANIZAÇÃO MUNDIAL DE SAÚDE - OMS. Assistência ao parto normal: um guia prático. Saúde materna e neonatal/ Unidade de maternidade segura. Saúde reprodutiva e da família. Tradução da Organização Panamericana de Saúde-OPAS. Genebra; 1996. 53p.

23. PROGIANTI JM, COSTA RF. A negociação do cuidado de enfermagem obstétrica através das práticas educativas na casa de parto. Esc Anna Nery Rev Enferm, dec 2008; 12(4): 789-92.

24. ZANETTI-DAELLENBACH RA, TSCHUDIN S, ZHONG XY et al. Maternal and neonatal infections and obstetrical outcome in water birth. Eur J Obstet Gynecol Reprod Biol, sep 2007; 134: 37-43. 